\title{
Tamoxifen Treatment and Risk of Endometrial Cancer:
}

\section{An Overview}

Fadila Kouhen, Fadoua Rais, Naoual Benhmidou, Mohammed Afif, Sanaa Elmajjaoui, Hanan Elkacemi, Tayeb Kebdani and Noureddine Benjaafar

Department of Radiotherapy, National Institute of Oncology, Mohammed Vuniversity in Rabat, Rabat, Morocco

\begin{abstract}
For several decades, tamoxifen has proven effective in reducing recurrence and mortality for early and metastatic breast cancer with positive estrogen receptors, and has been proven to be beneficial in preventing breast cancer in high-risk women. However, tamoxifen has been associated with an increased risk of endometrial cancer by its agonist effects on the endometrium in postmenopausal women. The purpose of this article, through an exhaustive summary of literature, is to provide a better understanding of this relationship and its impact on managed care on patients.
\end{abstract}

Key words: Tamoxifen, endometrial cancer, screening.

\section{Introduction}

Tamoxifen is a nonsteroidal antiestrogen discovered in 1960 during research on contraception, but the Food and Drug Administration approved it in 1978 for the treatment of patients with breast cancer with hormone receptor positive [1].

$\mathrm{He}$ has since been the subject of hundreds of scientific publications.

A large trial interesting 75,000 patients have demonstrated that tamoxifen improves disease-free survival as well as overall survival in patients with breast cancer with hormone receptor positive regardless of their menopausal status [2].

The clinical trial findings B14 reported by the National Surgical Adjuvant Breast and Bowel Project (NSABP) included 2,644 women with breast cancer without lymph node involvement and estrogen receptor positive [3]. They received $20 \mathrm{mg}$ of tamoxifen for 5 years. After 4 years, there was a significant prolongation of disease-free survival among women treated with tamoxifen, as compared with those receiving placebo ( $83 \%$ versus $77 \%$ ), but

Corresponding author: Fadila Kouhen, doctor, research field: radiation oncology. without significant benefit in overall survival. Multivariate analysis confirms the benefit regardless of age.

Several studies have shown that tamoxifen reduces also the incidence of occurrence of contralateral breast cancer $[1,4,5]$. This has prompted investigations to seek the interest of tamoxifen for prevention of breast cancer among high-risk women [6-8].

Endometrial cancer is the most common female genital cancer in Europe and North America with 54.870 new cases per year [9]. However, in developing countries, it is much less common than carcinoma of the cervix. In Morocco, it's the third gynecological cancer (after breast cancer and cervical cancer) and it concerns $2.7 \%$ of all cancers [10].

The large majority of patients are diagnosed at early stage of International Federation of Gynecology and Obstetrics [FIGO] with an overall survival of $95 \%$. The identified risk factors for endometrial cancer are numerous. Among the most important, obesity, diabetes, hypertensionand tamoxifen which is associated with small, but real, increased risk of endometrial cancer [11].

The first cases of endometrial cancer related to tamoxifen use were reported by Killackey in 1985 and 
since this association was the subject of several studies [12]. Currently, tamoxifenis considered as a risk factor of endometrial cancer.

In a study of the NSABP, the incidence of endometrial cancer in patients treated with tamoxifen $20 \mathrm{mg}$ per day was 1.6 per 1,000 per year versus 0.2 per 1,000 per year in patients in the control arm and the relative risk of endometrial cancer for the latter versus the former group was 7.5 [13]. However, net benefit greatly outweighs risk with a disease-free survival in the randomized tamoxifen group was $38 \%$ less than that in the placebo group.

The purpose of this article is to provide a better understanding of this relationship, and its impact on the care of patients. Our research was based on the articles and journals in English from the Medline, Cochrane library, Central, and Web of Science, with the following keywords alone or in combination: tamoxifen, cancer of the endometrium, hormone therapy.

\section{Pathophysiology}

Tamoxifen behaves as an estrogen antagonist or agonist depending on the target tissue and menopausal status of the patients $[14,15]$. While acting as an estrogen antagonistin the breast, it has estrogen agonist activity in other tissues; In the liver, its estrogenic actions predominates, reducingserum cholesterol levels with a favorable effect on the ratio of high density lipoprotein (HDL) versus low-density lipoprotein (LDL) cholesterol and a decrease in explaining its favorable cardiovascular effects. On bone, in premenopausal, tamoxifen has anti estrogenic actions while in postmenopausal, it has estrogenic action that promotes bone mineralization.

Finally, tamoxifen havean agonist effect on the endometriumthat can stimulate proliferation, which increases the risk of polyps, hyperplasia, and endometrial cancer in postmenopausal patients.

Tamoxifen is metabolized in the liver by the cytochrome P450 isoform CYP2D6 and CYP3A4 to active metabolites such as the 4-hydroxy and N-desmethyl-4-hydroxy (endoxifen) which have a much higher affinity to estrogen receptor than tamoxifen. These metabolites have the ability to form proteins or adducts, causing DNA damage [16]. Therefore, it was suggested that tamoxifen causes malignant tumors by genotoxicity.

The mechanism of action of tamoxifen is very complex. The ligand binding induce conformational change of the receptor that differs between oestrogen and tamoxifen [17]. The tissue-dependent mode of effect of tamoxifen can then be explained by the differential recruitment of co-factors in different tissues [18].

In breast cells, tamoxifen recruit the co-repressors: co-repressors nuclear receptor co-repressor (NCoR) and silencing mediator for retinoid and thyroid hormone receptors (SMRT) to the Estrogen Receptor (ER)-tamoxifen complex. However, in the endometrium the co-activators steroid receptor co-activator-1 (SRC-1), amplified in breast cancer-1 (AIB1) and CREB-binding protein (CBP) are recruited.

Few studies comparing the gene expression profiles of tamoxifen-associated and sporadic endometrial cancers $[19,20]$.

In a study by Ferguson et al, there was no difference between the gene-expression profile for tamoxifen-induced cancers and non-tamoxifen-associated endometrial cancer [19]. However, other studies have shown the opposite [20].

In vitro, tamoxifen induces endometrial cells proliferation by the activation of several signaling pathways that promote cell proliferation, including activated protein kinase mitogen and growth factor related to insulin ( IGF1) [21].

Similarly, in vivo, tamoxifen causes an increase of cell proliferation, which results in a high Ki67 proliferation index, and a stimulation of the anti-apoptotic pathway (Fas, FasL and Bcl2) [22]. Several recent studies have suggested that the UPR and mTOR signaling pathways are activated in 
long-term tamoxifen exposure-induced endometrial cancer [20, 23, 24].

By knowing the mechanism of action of tamoxifen, many new drugs were recently tested to lower the risk of endometrial cancer for tamoxifen users as: ABT-737, a $\mathrm{BH} 3$ mimetic drug, targeting the $\mathrm{Bcl}-2$ protein, Bcl-xL and Bcl-w. The combined administration of ABT-737 with tamoxifen to mice for 10 days reduced the increase in endometrial thickness, possibly by promoting apoptosis. However, the molecular mechanism of BH3 mimetics effect of tamoxifen in blocking effects requires further investigation [25].

\subsection{The Impact of Duration of Tamoxifen Treatment}

The risk of developing endometrial cancer in patients treated with tamoxifen is dose and duration dependent [26-30].

The analysis of the ATLAS experiment on the interest of extending the period of use of adjuvant tamoxifen 5 years to 10 years in 12,894 patients between 1996 to 2005 has shown that the extension of its use reduces the risk of recurrence breast cancer for women with a considerable risk of relapse after completing five years of adjuvant endocrinal therapy especially in node positive cases and younger women (617 recurrences among 3,428 patients versus 711 among 3,418 patients in the control arm, $P=0.002$ ), mortality from breast cancer (331 deaths versus 397 deaths, $P=0.01$ ), but increases risk of development of endometrial cancer (116 versus 63 with a $P: 0.0002)$. Therefore the authors suggest the use of tamoxifen for 10 years increases the risk of developing endometrial cancer by $2 \%$ [29].

Similarly, ATTOM trial (Adjuvant Tamoxifen To offer more) including 6,953 patients in 176 centers in the UK to seek the benefit of extending the use of adjuvant tamoxifen to 10 years reported 102 cases of endometrial cancer versus 45 cases $(\mathrm{RR}=2.20$, $P<0.0001)$, and higher rates of endometrial cancer death $(1.1 \%$ versus $0.6 \%, P=0.02)$ [30].

ATAC trial suggests that tamoxifen treatment causes a doubling of the risk of endometrial cancer after 1-2 years and a quadrupling after 5 years of therapy respectively [31].

\subsection{The Characteristics and Prognosis of Endometrial Cancer Induced by Tamoxifen}

Several studies have reported that the use of tamoxifen is associated with a higher incidence of uterine sarcomas and otherhigh-risk histologies (papillary serous, clear cell, mixed mesodermal tumor) [1, 32-35].

In a review of all the NASBP trials (B-09, B-14, B-21, B-23, B-24, and P-1), the incidence of uterine sarcomas in patients treated with tamoxifen was 10 per 10,000 per year versus no patient in the control arm [36].

Anthony et al reported a case-control study that the risk of developing malignant mixed mullerian tumors and sarcomas in patients on tamoxifen $(\mathrm{OR}=13.5,95 \%$ $\mathrm{CI}=4.1$ to 44.5$)$ was higher than the risk of adenocarcinoma $(\mathrm{OR}=2.1,95 \% \mathrm{CI}=1.6$ to 2.7$)$ [37].

However, other studies found no difference between endometrial cancers that develop after tamoxifen therapy and endometrial cancers occurring in the general population [38, 39].

In a study from the Memorial Sloan Kettering Cancer Center including 73 patients with breast cancer who developed endometrial cancer, no difference was found in terms of age, stage, histologic type of uterine cancer, or in survival in patients treated with tamoxifen compared with other patients [39].

Most authors suggest that the prognosis of tamoxifen endometrial cancer is often poorer than among non-users [40-42]. Hoogendoorn et al showed that the 3-year survival of specific endometrial cancer is lower compared to the control arm (82\% versus $93 \%, P=0.001)$ even after adjustment for age, histological type and stage [41].

A recent study by Institut Curie has analyzed patients treated for an endometrial carcinoma from 1994 to 2004: patients without breast cancer (Group 
1), patients with breast cancer without tamoxifen (Group 2) and patients with breast cancer with tamoxifen (group 3). Overall survival at 5 years was respectively in 1,2 and $3: 82 \%, 73.2 \%$ and $61 \%(P=$ $0.0006)$ [42].

\subsection{The Value of Screening Endometrial Cancer Treated with Tamoxifen}

\subsubsection{The Transvaginal Ultrasonography}

Transvaginal ultrasonography (TVS) is the initial examination for detection of endometrial abnormalities with a sensitivity of $90 \%$ and a specificity of $48 \%$ [43].

The definition of an abnormal endometrial stripe in the patient receiving tamoxifen for breast cancer remains unclear.

Jindal et al reported that the thickness of the endometrial lining more than $5 \mathrm{~mm}$ is sufficient to define an abnormal endometrial ultrasound scan [44].

Kedar et al reported that an endometrial thickness greater than $8 \mathrm{~mm}$ has a predictive value of $100 \%$ of the presence of endometrial abnormalities [45]. The thickness of the endometrial lining was greater than $5 \mathrm{~mm}$.

A cohort study had as objective to determine the prevalence and significance of endometrial changes in patients using tamoxifen with a six-monthly TVS was done and fractional curettage was performed if endometrial thickness $\geq 15 \mathrm{~mm}$ [46]. Only 12 patients $(11.6 \%)$ had endometrial thickness $\geq 15 \mathrm{~mm}$. Two patients had suspected malignancy on biopsy curettage which one was an adenosarcoma.

The conclusion of the study was that there's no evidence of the interest of a systematic screening of asymptomatic endometrial cancer in patients treated with tamoxifen and that the biopsy endometrial curettage must be reserved for patients with breakthrough bleeding or vaginal discharge.

\section{Endometrial Biopsy}

Barakat presented the results of a prospective study on the value of screening for endometrial abnormalities by endometrial biopsy in patients with breast cancer under tamoxifen on the 35th Congress of the American Society of Medical Oncology [47].

One hundred fifty-nine patients with a median age of 51 years were included. Endometrial biopsies were performed every 6 months during the first two years and then every year for the next three years, with 635 endometrial biopsies (an average of 5.8 per patient). Three patients had a hysterectomy but only a patient had a leiomyosarcoma.

The authors concluded that there is no interest of routine biopsy for screening in tamoxifen-treated women.

A more recent Indian study was performed in 50 patients treated for breast cancer with tamoxifen [44]. A transvaginal ultrasound in the follow-up was performed in all patients. If endometrial thickness was more than $5 \mathrm{~mm}$ hysteroscopy and endometrial biopsy was done.

Eleven patients (22\%) had an endometrial thickness between 5.1 to $10 \mathrm{~mm}$ and 2 patients (4\%) had an endometrial thickness more than $20 \mathrm{~mm}$. Hysteroscopy was performed in 11 patients. 3 patients had abnormal appearance but only one patient had an endometrial adenocarcinoma histologically confirmed.

However, other studies have proposed to classify patients into low risk and high risk groups of developing endometrial cancer based on the presence or absence of benign endometrial polyps before starting treatment with tamoxifen in patients with breast cancer [48].

In a study by So Berliereand colleagues, 264 postmenopausal breast cancer patients, candidates for treatment with tamoxifen were studied prospectively with pelvic ultrasonography. Forty-six women (17.4\%) had asymptomatic endometrial lesions diagnosed before starting tamoxifen, which were resected. The incidence of atypical hyperplasia was significantly higher in patients with abnormal lesion before treatment than in those without. 
Thus, as recommended by the National Comprehensive Cancer Network 2015, all patients taking tamoxifen treatment should have an annual gynecological exam. The trans-vaginal ultrasound with or without an endometrial samples should be reserved for patients who have vaginal bleeding [49].

The American College of Ostertricians and Gynecologists, the American Cancer Society and the Canadian Cancer Society also does not recommend routine screening of asymptomatic patients for endometrial cancer [50-52].

\section{Conclusions}

Although the risks of tamoxifen are greatly outweighed by its benefits, patients must be informed of the risk of hyperplasia and endometrial cancer and the necessity to alert their physician about any sign of abnormal vaginal bleeding.

There is no consensus for monitoring patients taking tamoxifen. Routine endometrial surveillance has not proved to be effective and can cause unnecessary intervention and anxiety.

\section{Conflict of Interest}

The authors declare that they have no competing interests.

\section{References}

[1] Freedman, O. C., Fletcher, G. G., and Gandhi, S., et al. 2015. "Adjuvant Endocrine Therapy for Early Breast Cancer: a Systematic Review of the Evidence for the 2014 Cancer Care Ontario Systemic Therapy Guideline." Curr. Oncol. 22 (1): S95-113. doi: 10.3747/co.22.2326.

[2] Early Breast Cancer Trialists' Collaborative Group (EBCTCG) 2005. "Effects of Chemotherapy and Hormonal Therapy for Early Breast Cancer on Recurrence and 15-year Survival: an Overview of the Randomised Trials.” Lancet 365 (9472): 1687-717.

[3] Fisher, B., Costantino, J., and Redmond, C., et al. 1989. "A Randomized Clinical Trial Evaluating Tamoxifen in the Treatment of Patients with Node-negative Breast Cancer who have Estrogen-receptor-positivetumors." $N$. Engl. J. Med. 320 (8): 479-84.

[4] Early Breast Cancer Trialists' Collaborative Group (EBCTCG). Davies, C., Godwin, J., and Gray, R., et al.
2011. "Relevance of Breast Cancer Hormone Receptors and Other Factors to the Efficacy of Adjuvant Tamoxifen: Patient-level Meta-analysis of Randomised Trials." Lancet 378 (9793): 771-84.

[5] Fisher, B., and Redmond, C. 1991. "New Perspective on Cancer of the Contralateral Breast: a Marker for Assessing Tamoxifen as a Preventive Agent." J. Natl. Cancer Inst. 83 (18): 1278-80.

[6] Vogel, V. G., Costantino, J. P., and Wickerham, D. L., et al. 2010. "Update of the National Surgical Adjuvant Breast and Bowel Project Study of Tamoxifen and Raloxifene (STAR) P-2 Trial: Preventing Breast Cancer." Cancer Prev. Res. (Phila) 3 (6): 696-706.

[7] Cuzick, J., Sestak, I., and Cawthorn, S. 2015. "Tamoxifen for Prevention of Breast Cancer: Extended Long-term Follow-up of the IBIS-I Breast Cancer Prevention Trial." Lancet Oncol. 16 (1): 67-75.

[8] Melnikow, J., Kuenneth, C., and Helms, L. J., et al. 2006. "Chemoprevention: Drug Pricing and Mortality: the Case of Tamoxifen." Cancer 107 (5): 950-8.

[9] Rebecca, L., Siegel, M. P. H., and Kimberly, D., et al. 2015. "Cancer Statistics." Ca Cancer J. Clin. 65 (1): 5-29.

[10] Tazi, M. A., Erraki, A., and Benjaafar, N. 2013. "Cancer Incidence in Rabat, Morocco: 2006-2008.” E. Cancer Medical Science 7: 338.

[11] Iqbal, J., Ginsburg, O. M., and Wijeratne, T. D., et al. 2012. "Endometrial Cancer and Venous Thromboembolism in Women under Age 50 who Take Tamoxifen for Prevention of Breast Cancer: a Systematic Review." Cancer Treat Rev. 38 (4): 318-28.

[12] Killackey, M. A., Hakes, T. B., and Pierce, V. K. 1985. "Endometrial Adenocarcinoma in Breast Cancer Patients Receiving Anti-estrogens." Cancer Treat Report 69 (2): 237-8.

[13] Fisher, B., Costantino, I. P., and Redmond, C. K., et al. 1994. "Endometrial Cancer in Tamoxifentreated Breast Cancer Patients: Findings from the National Surgical Adjuvant Breast and Bowel Project (NSABP) B-14." J. Natl. Cancer Inst. 86 (7): 527-37.

[14] Shang, Y., and Brown, M. 2002. "Molecular Determinants for the Tissue Specificity of SERMs." Science 295 (5564): 2465-8.

[15] Jouhadi, H., Kone, A. S., and Benchekroun, N., et al. 2009. "Tamoxifène et cancer de l'endomètre : à propos de dix cas." Revue Marocaine du Cancer 1 (1): 38-42.

[16] Hu, R., Hilakivi-Clarke, L., and Clarke, R. 2015. "Molecular Mechanisms of Tamoxifen-associated Endometrial Cancer (Review)." Oncol. Lett. 9 (4): 1495-501.

[17] Brzozowski, A. M., Pike, A. C., and Dauter, Z., et al. 1997. "Molecular Basis of Agonism and Antagonism in 
the Oestrogen Receptor.” Nature 389 (6652): 753-8.

[18] McDonnell, D. P. 2004. "The Molecular Determinants of Estrogen Receptor Pharmacology." Maturitas 48 (1): S7-12.

[19] Ferguson, S. E., Olshen, A. B., and Viale, A., et al. 2004. "Gene Expression Profiling of Tamoxifen-associated Uterine Cancers: Evidence for Two Molecular Classes of Endometrial Carcinoma." Gynecol. Oncol. 92 (2): 719-25.

[20] Gielen, S. C., Kühne, L. C., and Ewing, P. C., et al. 2005. "Tamoxifen Treatment for Breast Cancer Enforces a Distinct Gene-expression Profile on the Human Endometrium: an Exploratory Study." Endocr. Relat. Cancer 12 (4): 1037-49.

[21] Shang, Y., and Brown, M. 2002. "Molecular Determinants for the Tissue Specificity of SERMs." Science 295 (5564): 2465-8.

[22] Mourits, M. J., Hollema, H., and De Vries, E. G. 2002. "Apoptosis and Apop-tosis-associated Parameters in Relation to Tamoxifen Exposure in Postmenopausal Endometrium." Hum. Pathol. 33 (3): 341-6.

[23] Andersson, H., Helmestam, M., and Zebrowska, A., et al. 2010. "Tamoxifen-induced Adduct Formation and Cell Stress in Human Endometrial Glands." Drug. Metab. Dispos. 38 (1): 200-7.

[24] Erdemoglu, E., Güney, M., and Take, G., et al. 2009. "RAD001 (Everolimus) Can Prevent Tamoxifen-related Endo-metrial and Stromal Hyperplasia." Int. J. Gynecol. Cancer 19 (3): 375-9.

[25] Vaillant, F., Merino, D., and Lee, L., et al. 2013. "Targeting BCL-2 with the BH3 Mimetic ABT-199 in Estrogen Receptor-positive Breast Cancer." Cancer Cell 24 (1): 120-9.

[26] Chen, J. Y., Kuo, S. J., and Liaw, Y. P., et al. 2014. "Endometrial Cancer Incidence in Breast Cancer Patients Correlating with Age and Duration of Tamoxifen Use: a Population Based Study.” J. Cancer 5 (2): 151-5.

[27] Lindahl, B., Andolf, E., and Ingvar, C., et al .2008. "Adjuvant Tamoxifen in Breast Cancer Patients Affects the Endometrium by Time, an Effect Remaining Years after End of Treatment and Results in an Increased Frequency of Endometrial Carcinoma." Anticancer Res. 28 (2B): 1259-62.

[28] Mignotte, H., Lasset, C., and Bonadona, V., et al. 1998. "Iatrogenic Risks of Endometrial Carcinoma after Treatment for Breast Cancer in a Large French Case Control Study.” Int. J. Cancer 76 (3): 325-30.

[29] Davies, C., Pan, H., and Godwin, J., et al. 2013. "Long-term Effects of Continuing Adjuvant Tamoxifen to 10 years versus Stopping at 5 years after Diagnosis of Oestrogen Receptor-positive Breast Cancer: ATLAS, a Randomised Trial." Lancet 381 (9869): 805-16.
[30] Gray, R. G., Rea, D., and Handley, K., et al. 2013. "aTTom: Long-term Effects of Continuing Adjuvant Tamoxifen to 10 Years versus Stopping at 5 Years in 6,953 Women with Early Breast Cancer." J. Clin. Oncol. 31 (5).

[31] Gerber, B., Krause, A., and Reimer, T., et al. 2006. "Anastrazole versus Tamoxifen Treatment in Postmenopausal Women with Endocrine-responsive Breast Cancer and Tamoxifen-induced Endometrial Pathology." Clin. Cancer Res. 12 (4): 1245-50.

[32] Soo Yeon, C., Deuk Jae, S., and Na Yeon, H., et al. 2015. "MR Imaging Findings of Tamoxifen-associated Uterine Adenosarcoma: Report of Two Cases." Investig. Magn. Reson. Imaging. 19 (1): 56-61.

[33] Wysowski, D. K., Honig, S. F., and Beitz, J. 2002. "Uterine Sarcoma Associated with Tamoxifen Use." $N$. Engl. J. Med. 346 (23): 1832-3.

[34] Bland, A. E., Calingaert, B., and Secord, A. A., et al. 2009. "Relationship between Tamoxifen Use and High Risk Endometrial Cancer Histologic Types." Gynecol. Oncol. 112 (1): 150-4.

[35] Fles, R., Hoogendoorn, W. E., and Platteel, I., et al. 2010. "Genomic Profile of Endometrial Tumors depends on Morphological Subtype, Notontamoxifenexposure." Genes Chromosomes Cancer 49 (8): 699-710.

[36] Barakat, R. R., Wong, G., and Curtin, J. P., et al .1994. "Tamoxifen Use in Breast Cancer Patients who Subsequently Develop Corpus Cancer is not Associated with a Higher Incidence of Adverse Histologic Features." Gynecol. Oncol. 55 (2): 164-8.

[37] Swerdlow, A. J., and Jones, M. E. 2005. "Tamoxifen Treatment for Breast Cancer and Risk of Endometrial Cancer: A Case-Control Study." J. Natl. Cancer Inst. 97 (5): 375-84.

[38] Slomovitz, B. M., Sun, C. C., and Ramirez, P. T., et al. 2004. "Does Tamoxifen Use Affect Prognosis in Breast Cancer Patients who Develop Endometrial Cancer?" Obstet. Gynecol. 104 (2): 255-60.

[39] Jones, M. E., van Leeuwen, F. E., and Hoogendoorn, W. E., et al. 2012. "Endometrial Cancer Survival after Breast Cancer in Relation to Tamoxifen Treatment: Pooled Results from Three Countries.” Breast Cancer Research 14: R91.

[40] Tergas, A. I., Buell-Gutbrod, R., and Gwin, K., et al. 2012. "Clinico-pathologic Comparison of Type II Endometrial Cancers based on Tamoxifenexposure." Gynecol. Oncol. 127 (2): 316-20.

[41] Hoogendoorn, W. E., Hollema, H., and van Boven, H. H., et al. 2008. "Prognosis of Uterine Corpus Cancer after Tamoxifen Treatment for Breastcancer.” Breast Cancer Res. Treat 112 (1): 99-108.

[42] Ngô, C., Brugier, C., and Plancher, C., et al. 2014. 
"Clinico-pathology and Prognosis of Endometrial Cancer in Patients Previously Treated for Breast Cancer, with or without Tamoxifen: a Comparative Study in 363 Patients." Eur. J. Surg. Oncol. 40 (10): 1237-44.

[43] Genc, M., Genc, B., and Sahin, N., et al. 2015. "Endometrial Pathology in Postmenopausal Women with no Bleeding." Climacteric 18 (2): 241-5.

[44] Jindal, A., Manjit, K., and Mohi, M. K., et al. 2015. "Endometrial Evaluation by Ultrasonography, Hysteroscopy and Histopathology in Cases of Breast Carcinoma on Tamoxifen Therapy." Journal of Mid-life Health 6 (2): 59-65.

[45] Kedar, R. P., Bourne, T. H., and Powles, T. J., et al. 1994. "Effects of Tamoxifen on the Uterus and Ovaries of Postmenopausal Women in a Randomized Breast Cancer Prevention Trial." Lancet 343 (8909): 1318-21.

[46] Marcus, S., Mahajan, M. K., and Grover, S., et al. 2014. "Significance and Prevalence of Tamoxifene Related Endometrial Changes in Patients of Carcinoma Breast." International Journal of Scientific and Research
Publications 4 (3): 1-7.

[47] Barakat, R. R., Gilewski, T. A., and Almadrones, L., et al. 2000. "The Effect of Adjuvant Tamoxifen (T) on the Endometrium in Women with Breast Cancer: A Prospective Study." JCO 18 (20): 3459-63.

[48] Berliere, M., Charles, A., and Galant, C., et al. 1998. "Uterine Side Effects of Tamoxifen: a Need for Systematic Pretreatment Screening." Obstet. Gynecol. 91 (1): 40-4.

[49] National Comprehensive Cancer Network. 2015. NCCN Clinical Practice Guidelines in Oncology: Breast Cancer. V.2.2015. Accessed at www.nccn.org.

[50] Committee on Gynecologic of American College of Obstetricians and Gynecologists. 2006. "Tamoxifen and Uterine Cancer.” Obstetrics \& Gynecology 107: 1475-8.

[51] American Cancer Society. 2015. Cancer Facts \& Figures (2015). Atlanta, Ga.

[52] Canadian Cancer Society, National Cancer Institute of Canada. 2013. Canadian Cancer Statistics 2013. Toronto, ON. 